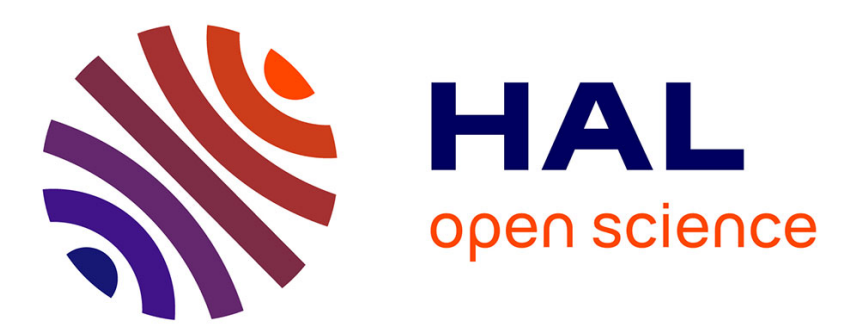

\title{
Direction-of-Arrival Estimation for CS-MIMO Radar Using Subspace Sparse Bayesian Learning
}

\author{
Yang Bin, Huang Dongmei, Li Ding
}

\section{To cite this version:}

Yang Bin, Huang Dongmei, Li Ding. Direction-of-Arrival Estimation for CS-MIMO Radar Using Subspace Sparse Bayesian Learning. 9th International Conference on Intelligent Information Processing (IIP), Nov 2016, Melbourne, VIC, Australia. pp.31-38, 10.1007/978-3-319-48390-0_4 . hal-01614998

\author{
HAL Id: hal-01614998 \\ https://hal.inria.fr/hal-01614998
}

Submitted on 11 Oct 2017

HAL is a multi-disciplinary open access archive for the deposit and dissemination of scientific research documents, whether they are published or not. The documents may come from teaching and research institutions in France or abroad, or from public or private research centers.
L'archive ouverte pluridisciplinaire HAL, est destinée au dépôt et à la diffusion de documents scientifiques de niveau recherche, publiés ou non, émanant des établissements d'enseignement et de recherche français ou étrangers, des laboratoires publics ou privés. 


\title{
Direction-of-Arrival Estimation for CS-MIMO radar using Subspace Sparse Bayesian Learning
}

\author{
Yang Bin ${ }^{1}$, Huang Dongmei ${ }^{2}$, Li Ding ${ }^{2}$ \\ 1 Shanxi Electric Power Technical College, Taiyuan, 030021, China \\ 2 Naval Command College, Nanjing, 210016, China \\ wfqsyyy@163.com
}

\begin{abstract}
We address the problem of direction-of-arrival (DOA) estimation for compressive sensing based multiple-input multiple-output (CS-MIMO) radar. The spatial sparsity of the targets enables CS to be desirable for DOA estimation. By discretizing the possible target angles, a overcomplete dictionary is constructed for DOA estimation. A structural sparsity Bayesian learning framework is presented for support recovery. To improve the recovery accuracy and speed up the Bayesian iteration, a subspace sparse Bayesian learning algorithm is developed. The proposed scheme, which needs less iteration steps, can provides high precision DOA estimation performance for CS-MIMO radar, even at the condition of low signal-to-noise ratio and coherent sources. Simulation results verify the usefulness of our scheme.
\end{abstract}

Keywords: multiple-input multiple-output radar; sparse Bayesian learning; angle estimation; subspace decomposition

\section{Introduction}

Multiple-input multiple-output (MIMO) radar is a relatively new concept for radar system. By exploiting multiple antennas in both transmit and receive end, the extra visual antenna aperture is formed. The visual aperture makes the performance of MIMO radar better than the traditional phased array radar [1]. Generally speaking, the array geometry in MIMO radar can be divided into two categories, the uniform array and the nonuniform array. Elements in the uniform array geometry must be spaced at intervals no larger than half wavelength of the carrier signal thus to avoid phase ambiguity. Typical uniform arrays including linear arrays, uniform circular array and L-shape or rectangle array. The nonuniform array setup is much more flexible than the uniform array configuration [2]. The minimum redundancy linear array and the random array are belong to this kind of array.

Direction-of-arrival (DOA) estimation is a fundamental problem in MIMO radar that has aroused extensive attention. Existing estimation algorithms including Capon [3], multiple signal classification (MUSIC) [4], the estimation method of signal parameters via rotational invariance techniques (ESPRIT) [5]-[7], the parallel factor analysis (PARAFAC) [8]-[9]. However, the majority of the above algorithms are effectiveless with the nonuniform arrays. Algorithm Capon and MUSIC are effective with nonuniform configuration, they only perform well with large number of snapshot. Besides, additional 
prior information is needed in this algorithm, such as the number of targets, the noise level, et al. Recently, compressive sensing (CS) theory has attracted extensive attention in the field of array signal processing[10]-[12]. In this paper, we focus on the compressive sensing based MIMO (CS-MIMO) radar [13]. In CS-MIMO radar, transmit and receive elements are randomly placed over a large aperture and spatial sampling is applied at sub-Nyquist rate. The random array setup would achieve similar resolution with significantly fewer elements. In fact, the targets in CS-MIMO can be viewed as sparse in the background. Therefore, DOA estimation can be regarded as a sparse inverse problem from multiple measurement vectors (MMV) in CS. Actually, there are many excellent algorithms for the MMV problem, such as Basis Pursuit (BP) [14], Orthogonal Matching Pursuit (OMP) [15], FOCal Underdetermined System Solver (FOCUSS) [16], Sparse Bayesian Learning (SBL) [17]. The sparse inverse problem makes the DOA estimation more accurate than the traditional methods. Unfortunately, the computational complexity of BP algorithm is too large to engineering implementation. Both OMP and FOCUSS are sensitivity to noise, and it is hard for FOCUSS to choose a proper regularization parameter. SBL is a blind recovery algorithm that always achieves the sparsest global minima. The statistical model that SBL based provides a flexible framework to exploit special structures in the model [18], which may significantly improve the recovery performance. A SBL based DOA estimation algorithm has been proposed for CS-MIMO radar [19], but it suffers from high computational complexity, making it unsuitable for the radar system.

In this paper, a structural correlated subspace SBL algorithm is derived for DOA estimation in CS-MIMO radar. The problem of DOA estimation is formulated as a sparse inverse problem. A statistical model is present which utilize the intra- and the extrainformation of the data. DOA estimation is linked to parameters iteration in the SBL model. To speed up the learning process, the subspace-based sparse Bayesian learning (S-SBL) algorithm is developed. The proposed algorithm can reduce the complexity of the recovery process and enable the radar system keep good performance in lower signalto-noise ratio (SNR), even with coherent sources. Experimental results show the proposed scheme performs better than the existing algorithms.

The paper outline is as follows. The data model for the CS-MIMO radar is presented in section 2. The proposed S-SBL algorithm is derived in section 3. Simulation results are given in section 4 . We end the paper by a brief concluding in section 5 .

Notation, capital letters and lower case in bold denote, respectively, matrices and vectors. The superscript $(\boldsymbol{X})^{T},(\boldsymbol{X})^{H},(\boldsymbol{X})^{-1}$ and $(\boldsymbol{X})^{\dagger}$ represent the operations of transpose, Hermitian transpose, inverse and pseudo-inverse, respectively; $\boldsymbol{X}_{k \bullet}$ and $\boldsymbol{X}_{\bullet}$ represent the $k$-th row and $k$-th column of $\boldsymbol{X}$. The subscript $\|\boldsymbol{X}\|_{F}$ denote the Frobenius norm of $\boldsymbol{X} ; \otimes$ stands for the Kronecker product; The Khatri-Rao product (column-wise Kronecker product) is denoted by $\odot$, i.e., $\left[\boldsymbol{a}_{1}, \cdots, \boldsymbol{a}_{K}\right] \odot\left[\boldsymbol{b}_{1}, \cdots, \boldsymbol{b}_{K}\right]=\left[\boldsymbol{a}_{1} \otimes \boldsymbol{b}_{1}, \cdots, \boldsymbol{a}_{K} \otimes \boldsymbol{b}_{K}\right]$; The $M \times M$ identity matrix is denoted by $\boldsymbol{I}_{M}$, and the $M \times M$ inverse permutation matrix is denoted by $\boldsymbol{I}_{M}$. 


\section{Signal Model}

Consider the model for monostatic CS-MIMO radar in Figure 1[13]. The radar system is consist of $M$ transmit elements and $N$ receive elements. Both transmit elements and receive elements are located in the $X$-axis. The total transmit aperture and the receive aperture is $Z_{T X}$ time and $Z_{R X}$ time of the carrier signal wavelength, respectively. The position of the $m$-th transmitter and the $n$-th receiver is denoted by $\frac{Z}{2} \zeta_{m}$ and $\frac{Z}{2} \xi_{n}$, respectively. Define $Z=Z_{T X}+Z_{R X}$, with $\zeta_{m}$ lies in the interval $\left[-\frac{Z_{T X}}{Z}, \frac{Z_{T X}}{Z}\right]$ and $\xi_{n}$ lies in the interval $\left[-\frac{Z_{R X}}{Z}, \frac{Z_{R X}}{Z}\right]$. Suppose that the transmit elements' locations $\zeta$ are independent and identically distributed (i.i.d.) random variables governed by a distribution $p(\zeta)$. Similarly, the positions of the receivers $\xi$ be drawn i.i.d. from a distribution $p(\xi)$. We assumed that there are $K$ targets appearing in the far-field of the CS-MIMO radar system, the DOA of the $k$-th target is denoted by $\theta_{k}$. Supposed that the transmit elements emit ideal orthogonal waveforms. In the receive end, the matched filters are used to separate the information from each visual transmitter-to-receiver path. The output of the matched filters with sampled $L$ snapshots can be expressed as

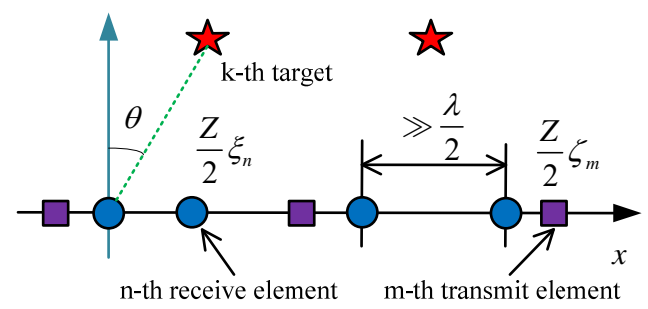

Fig. 1. Signal model of DOA estimation for CS-MIMO radar

$$
\boldsymbol{Y}=\left[\boldsymbol{A}_{T}(\theta) \odot \boldsymbol{A}_{R}(\theta)\right] \boldsymbol{S}+\boldsymbol{N}=\boldsymbol{A S}+\boldsymbol{N}
$$

where $\boldsymbol{A}_{T}(\theta)=\left[\boldsymbol{a}_{t}\left(\theta_{1}\right), \boldsymbol{a}_{t}\left(\theta_{2}\right), \cdots, \boldsymbol{a}_{t}\left(\theta_{K}\right)\right] \in \mathbb{C}^{M \times K} \quad$ is the transmit direction matrix, with the $k$-th $(k=1,2, \cdots, K)$ transmit steering vector $\boldsymbol{a}_{t}\left(\theta_{k}\right)$ is denoted by $\boldsymbol{a}_{t}\left(\theta_{k}\right)=\left[\exp \left(j \pi Z \zeta_{1} \sin \theta_{k}\right), \exp \left(j \pi Z \zeta_{2} \sin \theta_{k}\right) \cdots, \exp \left(j \pi Z \zeta_{M} \sin \theta_{k}\right)\right]^{T} \in \mathbb{C}^{M \times 1}$ $\boldsymbol{A}_{R}(\theta)=\left[\boldsymbol{a}_{r}\left(\theta_{1}\right), \boldsymbol{a}_{r}\left(\theta_{2}\right), \cdots, \boldsymbol{a}_{r}\left(\theta_{K}\right)\right] \in \mathbb{C}^{N \times K}$ is the receive direction matrix, and the $k$-th $(k=1,2, \cdots, K)$ receive steering vector $\boldsymbol{a}_{r}\left(\theta_{k}\right)$ is given by $\boldsymbol{a}_{r}\left(\theta_{k}\right)=\left[\exp \left(j \pi Z \xi_{1} \sin \theta_{k}\right), \exp \left(j \pi Z \xi_{2} \sin \theta_{k}\right) \cdots, \exp \left(j \pi Z \xi_{N} \sin \theta_{k}\right)\right]^{T} \in \mathbb{C}^{N \times 1}$. The visual direction matrix is $\boldsymbol{A}=\left[\boldsymbol{A}_{T}(\theta) \odot \boldsymbol{A}_{R}(\theta)\right] \in \mathbb{C}^{M N \times K} . \boldsymbol{S} \in \mathbb{C}^{K \times L}$ is the source matrix and $N \in \mathbb{C}^{M N \times L}$ represent an additive Gaussian white noise matrix with variance $\sigma_{N}^{2}$.

Additional assumption is that the sources are located in the maximum unambiguous angles. By discretizing the possible angle on a fine uniform grid, i.e., $\left[\varphi_{1}, \varphi_{2} \cdots, \varphi_{G}\right]$ ( $K \ll G)$, we can obtain an visual overcomplete dictionary 
$\boldsymbol{B}=\left[\boldsymbol{b}\left(\varphi_{1}\right), \boldsymbol{b}\left(\varphi_{2}\right) \cdots, \boldsymbol{b}\left(\varphi_{G}\right)\right] \in \mathrm{C}^{M N \times G}$ for DOA estimation, where the $g$-th $(g=1,2 \cdots, G)$ steering vector $\boldsymbol{b}\left(\varphi_{g}\right)=\boldsymbol{a}_{t}\left(\varphi_{g}\right) \otimes \boldsymbol{a}_{r}\left(\varphi_{g}\right)$, with $\boldsymbol{a}_{t}\left(\varphi_{g}\right)$ is $\boldsymbol{a}_{t}\left(\varphi_{g}\right)=\left[\exp \left(j \pi Z \zeta_{1} \sin \varphi_{g}\right), \exp \left(j \pi Z \zeta_{2} \sin \varphi_{g}\right) \cdots, \exp \left(j \pi Z \zeta_{M} \sin \varphi_{g}\right)\right]^{T} \in \mathbb{C}^{M \times 1} \quad$, and $\boldsymbol{a}_{r}\left(\varphi_{g}\right)=\left[\exp \left(j 2 \pi Z \xi_{1} \sin \varphi_{g}\right), \exp \left(j 2 \pi Z \xi_{2} \sin \varphi_{g}\right) \cdots, \exp \left(j 2 \pi Z \xi_{M} \sin \varphi_{g}\right)\right]^{T} \in \mathbb{C}^{N \times 1}$. Therefore, the received data in (1) can be written as

$$
\boldsymbol{Y}=\boldsymbol{B} \boldsymbol{X}+\boldsymbol{N}, \quad \boldsymbol{X}_{g \bullet}=\left\{\begin{aligned}
\boldsymbol{S}_{k \bullet}, & \text { when } \varphi_{g}=\theta_{k}, \\
\mathbf{0}, & \text { others }
\end{aligned}\right.
$$

Noting that $K \ll M N<G$, the model in (2) is a MMV problem [16]. The DOA estimation problem can be expressed as to recover $\boldsymbol{\theta}$ from the given measurement matrix $\boldsymbol{Y}$ and the known matrix $\boldsymbol{B}$, which is equal to recovery the support $\operatorname{supp}(\boldsymbol{X})$ (non-zero rows of $\boldsymbol{X}$ ). The previous work in [13] has shown that once $M N$ satisfies certain boundary condition, $\boldsymbol{B}$ would provide a uniform recovery guarantee for(2). Relying on a random array geometry, the model in (2) is linked to the CS framework.

\section{Environment Awareness}

The subspace that obtained from the covariance matrix decomposition can improve the DOA estimation accuracy in low SNR scene. The covariance matrix of the received data can be estimated by $\hat{\boldsymbol{R}}_{Y}=\boldsymbol{Y} \boldsymbol{Y}^{H} / L$. If the sources are noncoherent, the rank of $\boldsymbol{R}_{Y}$ is $\operatorname{rank}\left(\boldsymbol{R}_{Y}\right)=K$. But there is a rank loss if the sources are coherent. To maintain the rank of $\boldsymbol{R}_{Y}$, the spatial smooth method is applied to $\boldsymbol{R}_{Y}$. The estimated covariance matrix becomes

The eigenvalue decomposition of $\boldsymbol{R}$ is

$$
\boldsymbol{R}=\boldsymbol{R}_{Y}+\boldsymbol{I I}_{M N} \boldsymbol{R}_{Y}^{*} \boldsymbol{I}_{M N}
$$

$$
\boldsymbol{R}=\boldsymbol{U} \boldsymbol{\Sigma} \boldsymbol{U}^{H}=\sum_{j=1}^{M N} \lambda_{j} \boldsymbol{u}_{j} \boldsymbol{u}_{j}^{H}=\boldsymbol{U}_{s} \boldsymbol{\Sigma}_{s} \boldsymbol{U}_{s}^{H}+\boldsymbol{U}_{n} \boldsymbol{\Sigma}_{n} \boldsymbol{U}_{n}^{H}
$$

where $\boldsymbol{\Sigma}=\operatorname{diag}\left(\lambda_{1}, \lambda_{2}, \cdots, \lambda_{M N}\right)$, the eigenvalues are complied with $\lambda_{1} \geq \cdots \geq \lambda_{K}>$ $\lambda_{K+1}=\cdots=\lambda_{M N}=\sigma_{N}^{2}$. The eigenvectors $\boldsymbol{u}_{1}, \boldsymbol{u}_{2}, \cdots, \boldsymbol{u}_{k}$ corresponding to the $K$ larger eigenvalues $\lambda_{1}, \lambda_{2}, \cdots, \lambda_{N}$ construct signal subspace $\boldsymbol{U}_{s}=\left[\boldsymbol{u}_{1}, \boldsymbol{u}_{2}, \cdots, \boldsymbol{u}_{k}\right]$, with $\Sigma_{s}=\left[\lambda_{1}, \lambda_{2}, \cdots, \lambda_{K}\right]$. Similarly, the later $M N-K$ eigenvalue are depending on the noise and their numeric values are $\sigma_{N}^{2}$. The eigenvectors $\boldsymbol{u}_{K+1}, \boldsymbol{u}_{K+2}, \cdots, \boldsymbol{u}_{M N}$ corresponding to $\lambda_{K+1}, \lambda_{K+2}, \cdots, \lambda_{M N}$ construct noise subspace $\boldsymbol{U}_{n}=\left[\boldsymbol{u}_{K+1}, \boldsymbol{u}_{K+2}, \cdots, \boldsymbol{u}_{N}\right]$, and $\Sigma_{n}=\left[\lambda_{K+1}, \lambda_{K+2}, \cdots, \lambda_{N}\right]$. Generally, the number of the sources can be estimated by the distribution of the eigenvalue. Let $\boldsymbol{Z}=\boldsymbol{U}_{s} \in \mathbb{C}^{M N \times K}$ denotes the estimated signal subspace, hence there exist a nonsingular matrix $\boldsymbol{T} \in \mathbb{C}^{M N \times M N}$ that

$$
\boldsymbol{Z}=\boldsymbol{B} \boldsymbol{T}
$$


Note that the dimension of $\boldsymbol{Z}$ is much smaller than $\boldsymbol{Y}$, therefore the required iteration steps of SBL for $\boldsymbol{Z}$ will less than $\boldsymbol{Y}$. Let $\lambda$ be the variance parameter of the noise, $\gamma_{g}$ is a hyperparameter, $\boldsymbol{R}$ is the covariance matrix of $\mathbf{X}_{g}$, which captures the intra-block correlation of the source. The mean and covariance parameters are given by $\boldsymbol{\mu}$ and $\boldsymbol{\Sigma}$. According to [18], the $d+1$-th iteration process of the structural correlated SBL algorithm can be summarized as follows

$$
\left\{\begin{array}{l}
\left(\gamma_{g}\right)_{d+1}=\frac{1}{L}\left(\boldsymbol{X}_{g \bullet}\right)_{d} \boldsymbol{R}_{d}^{-1}\left(\boldsymbol{X}_{g \bullet}^{T}\right)_{d}+\left(\left(\Sigma_{\mathbf{X}}\right)_{d}\right)_{g g}, \quad g=1,2 \cdots, G \\
\boldsymbol{R}_{d+1}=\tilde{\boldsymbol{R}}_{d+1} /\left\|\tilde{\boldsymbol{R}}_{d+1}\right\|_{F}, \quad, \quad \tilde{\boldsymbol{R}}_{d+1}=\sum_{g=1}^{G}\left(\boldsymbol{X}_{g \bullet}^{T}\right)_{d}\left(\boldsymbol{X}_{g \bullet}\right)_{d}+\eta \boldsymbol{I} \\
\lambda_{d+1}=\frac{1}{M N L}\left\|\boldsymbol{Z}-\boldsymbol{B} \boldsymbol{X}_{d}\right\|_{F}^{2}+\frac{\lambda_{d}}{G} \operatorname{Tr}\left[\boldsymbol{B} \boldsymbol{H}_{d}\right] \\
\left(\Sigma_{\mathbf{X}}\right)_{d+1}=\left(\boldsymbol{\Lambda}_{d}^{-1}+\frac{1}{\lambda_{d}} \boldsymbol{B}^{T} \boldsymbol{\Lambda}_{d}\right), \quad \boldsymbol{\Lambda}=\operatorname{diag}\left[\gamma_{1}, \gamma_{2}, \cdots, \gamma_{G}\right] \\
\boldsymbol{X}_{d+1}=\boldsymbol{H}_{d} \boldsymbol{Z}, \quad \boldsymbol{H}_{d}=\boldsymbol{\Lambda}_{d} \boldsymbol{B}^{T}\left(\lambda_{d} \boldsymbol{I}+\boldsymbol{B} \boldsymbol{\Lambda}_{d} \boldsymbol{B}^{T}\right)^{-1}
\end{array}\right.
$$

where $\eta$ is a positive scalar. With the iteration of the SBL, the matrixes are trend to stable. The iteration will repeat until algorithm convergence thus we obtain the estimated $\hat{\boldsymbol{X}}$. With the non-zeros rows in $\hat{\boldsymbol{X}}$ we can get the estimated DOAs.

\section{Simulation results}

In this section, 1000 Monte Carlo trails are used to assess the DOA estimation performance of the S-SBL algorithm. In our simulation, the successful-rate and average running time are used for performance assessment. A successful trial was recognized if the indexes of estimated sources with $K$ largest $l_{2}$-norms were the support of $\boldsymbol{X}$. Parameters in our simulation are set to $Z=250, M=N=6, \boldsymbol{\theta}=[15,40,65]$. The range of the angle in the dictionary is $\left[0^{\circ}, 90^{\circ}\right]$ with interval $0.1^{\circ}$. The SNR in the simulation is defined as $10 \log \left(\|\boldsymbol{Y}-\boldsymbol{N}\|_{2}^{2} /\|\boldsymbol{N}\|_{2}^{2}\right)$. We compared our algorithm with traditional MUSIC algorithm [4], the OMP method[15], the FOCUSS algorithm [16] and the SBL method[17].

Figure 2 and figure 3 depict the noncoherent sources estimation performance comparison of all the algorithms with different SNR, respectively. It can be seen from figure 2 that all algorithms would achieve better performance with the increasing SNR, while the S-SBL algorithm provides almost precise results. Another result is that the SSBL runs faster than the SBL method. 


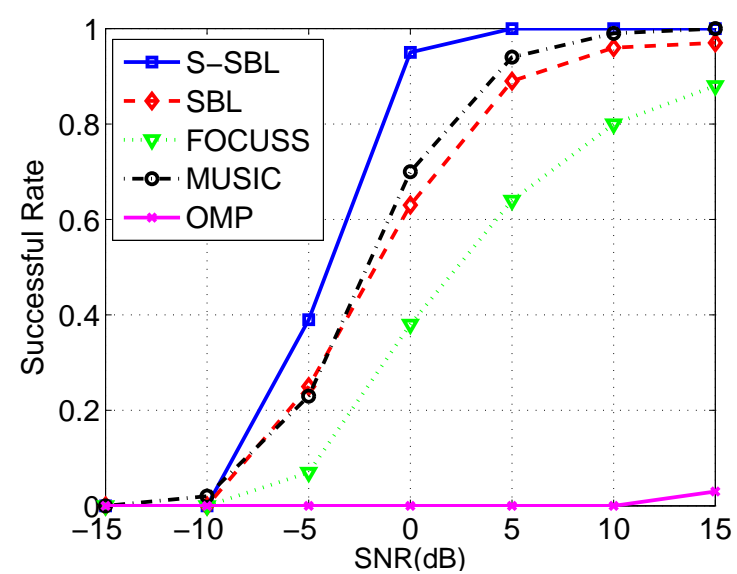

Fig. 2. Successful Rate comparison for noncoherent sources with different SNR

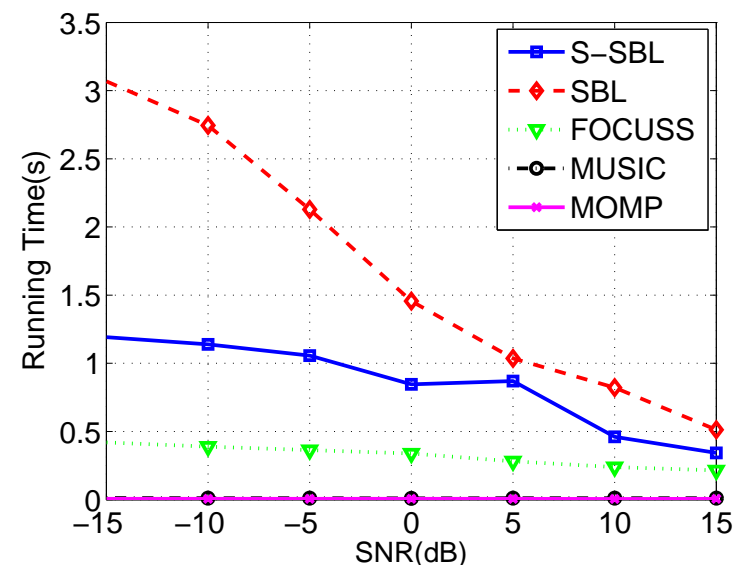

Fig. 3. Average running time comparison for noncoherent sources with different SNR

Figure 4 and figure 5 depict the coherent sources estimation performance comparison of all the algorithms with different SNR, respectively. In the simulation, the second target and the third target are coherent. It can be seen from the results that the Bayesian algorithms are outperform the other algorithms in this situation. Besides, the S-SBL method perform better than the SBL approach, and the S-SBL method requires less computational load than the SBL method. 


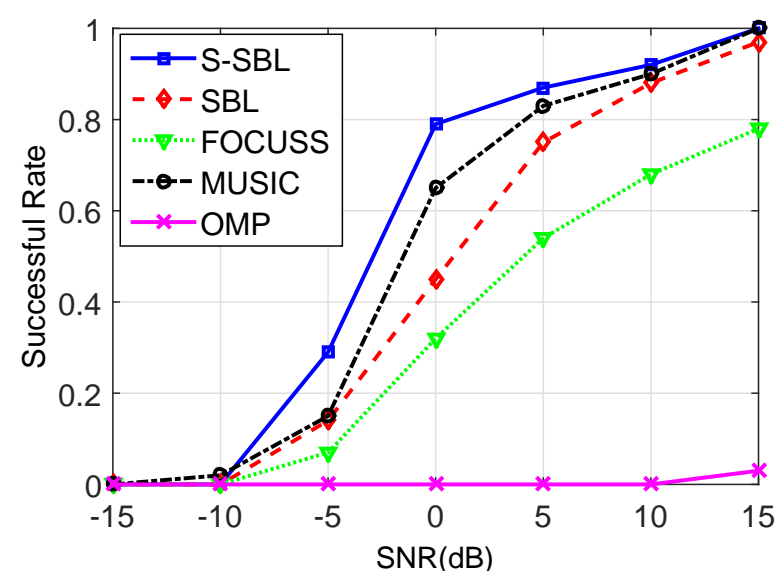

Fig. 4. Average running time comparison for coherent sources with different SNR

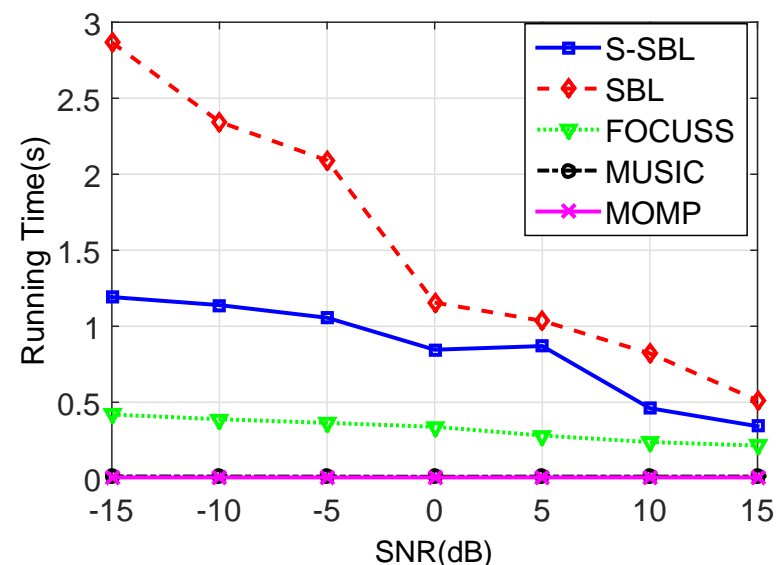

Fig. 5. Average running time comparison for coherent sources with different SNR

\section{Conclusions}

In this paper, we have proposed a subspace-based SBL algorithm for DOA estimation in CS-MIMO radar. The subspace operation deduces the computational load and improves the estimation accuracy in low SNR scene. Our work links the estimation of angle to the parameters learning in the Bayesian framework. The S-SBL method does not require the prior information of the number of targets, and outperforms the traditional MUSIC algorithm and existing CS recovery methods.

\section{References}

[1]. Fishler, E., Haimovich, A., Blum, R., Chizhik, D., Cimini, L., Valenzuela, R., 'MIMO radar: an idea whose time has come', Proc. IEEE Radar Conf., April 2004, pp. $71-78$ 
[2]. Wen F., Zhang G. Two-Dimensional Direction-of-Arrival Estimation for Trilinear Decomposition-Based Monostatic Cross MIMO Radar [J]. Mathematical Problems in Engineering, 2013, 427980.

[3]. Zhang, X., Huang, Y., Chen, C., Li, J., Xu, D., 'Reduced-complexity Capon for direction of arrival estimation in a monostatic multiple-input multiple-output radar', IET Radar Sonar Navig., 2012, 6, (8), pp. 796-801

[4]. Yan H., Li J., Liao G., 'Multitarget identification and localization using bistatic MIMO radar systems', EURASIP J. Adv. Signal Process. 2008, 2008: 48

[5]. Duofang, C., Baixiao, C., Guodong, Q., 'Angle estimation using ESPRIT in MIMO radar', Electron. Lett., 2008, 44, (12), pp.770-771

[6]. Jinli C, Hong G, Weimin S. 'Angle estimation using ESPRIT without pairing in MIMO radar', Electron. Lett., 2008, 44, (24): pp. 1422-1423

[7]. Zheng, Z.D., Zhang, J.Y., 'Fast method for multi-target localisation in bistatic MIMO radar', Electron. Lett., 2011, 47, (2), pp.138-139

[8]. Zhang, X., Xu, Z., Xu, L., Xu, D., 'Trilinear decomposition-based transmit angle and receive angle estimation for multiple-input multiple-output radar', IET Radar Sonar Navig., 2011, 5, (6), pp. 626-631

[9]. Li J, Zhou M, 'Improved trilinear decomposition-based method for angle estimation in multiple-input multiple-output radar', IET Radar Sonar Navig., 2013, 7, (9), pp.1019-1026

[10]. Wen F. Q., Tao Y., Zhang G. Analogue-to-information conversion using multicomparator-based integrate-and-fire sampler [J]. Electronics Letters. 2015, 51, (3), pp.246-247.

[11]. Wen F. Q., Zhang G., Ben D. Estimation of multipath parameters in wireless communications using multi-way compressive sensing [J]. Journal of Systems Engineering and Electronics, 2015, 26, (5), pp.908-915.

[12]. Wen F. Q., Zhang G., Ben D. Adaptive Selective Compressive Sensing based Signal Acquisition Oriented toward Strong Signal Noise Scene [J]. KSII Transactions on Internet and Information Systems, 2015, 9, (9), pp.3559-3571.

[13]. Rossi, M., Haimovich, A. M., \& Eldar, Y. C, Spatial compressive sensing for MIMO radar. Signal Processing, IEEE Transactions on, 2014, 62, (2), pp. 419-430.

[14]. Chen, S. S., Donoho, D. L., \& Saunders, M. A. Atomic decomposition by basis pursuit. SIAM review, 2001, 43 (1), pp. 129-159. 
[15]. Tropp, J. A., \& Gilbert, A. C. Signal recovery from random measurements via orthogonal matching pursuit. Information Theory, IEEE Transactions on, 2007, 53 (12), 4655-4666.

[16]. Cotter, S. F., Rao, B. D., Engan, K., \& Kreutz-Delgado, K. Sparse solutions to linear inverse problems with multiple measurement vectors. Signal Processing, IEEE Transactions on, 2005, 53, (7), 2477-2488.

[17]. Tipping, M. E. Sparse Bayesian learning and the relevance vector machine. The journal of machine learning research, 2001, 1, 211-244.

[18]. Wen F. Q., Zhang G., Ben D. A recovery algorithm for multitask compressive sensing based on block sparse Bayesian learning, 2015, 70201-070201.

[19]. Wen F. Q., Zhang G., Ben D. Direction-of-Arrival Estimation for Multiple-Input Multiple-Output Radar Using Structural Sparsity Bayesian Learning [J]. Chinese Physics B, 2015, 24, (11): 110201 